\title{
¿Sostenibilidad fuerte o débil? Perspectivas de la protección jurídico- ambiental y ecosistémica de los humedales. El caso del Partido de Lobos, Provincia de Buenos Aires, Argentina Strong or weak Sustainability? Environmental and ecosystem legal protection of wetlands. The case of the District of Lobos, Province of Buenos Aires, Argentina
}

\author{
Clara María Minaverry \\ Universidad de Buenos Aires, Argentina \\ cminaverry@derecho.uba.ar
}

Recibido: $18 / 04 / 2020$

Aceptado 22/09/2020

Resumen: En el presente trabajo se propone, en primer lugar, realizar un relevamiento de la normativa aplicable al Partido de Lobos, en la Provincia de Buenos Aires, Argentina, para luego poder contrastarla con la legislación vigente en otros países de América Latina. Luego de dicho análisis normativo se determinará dentro de qué categoría doctrinaria se encuentra la misma: Sustentabilidad "super-fuerte", "fuerte" o débil". En segundo lugar, se analizarán los resultados de las encuestas realizadas a la población urbana del Partido de Lobos y la información obtenida de entrevistas a informantes clave. Finalmente fue posible relevar que a menores niveles de educación y a mayor edad de los encuestados se demuestra más bajo interés y preocupación vinculados con problemáticas ambientales. Además, el desarrollo 
normativo es incipiente, en tanto existen escasas políticas públicas ambientales vinculadas con la Laguna de Lobos, a pesar de que implica un importante aporte económico proveniente del turismo.

Palabras claves: Derecho Ambiental, Humedales, Normativa, Sostenibilidad, Ecosistemas.

\begin{abstract}
The purpose of this paper is, in the first place, to review regulations valid for the District of Lobos, in the Province of Buenos Aires, Argentina, in order to contrast them with valid legislation of other countries of Latin America. After this regulatory analysys we will state in which doctrinaire category they will be included: "Super strong", "Strong" and "Weak" Sustainability. In the second place, we will analyze the results of surveys conducted in urban population of the District of Lobos, and the information provided by the interviews of key informants. Finally, we could state that people with lower educational levels and the older ones have less interest and concern about environmental problems. Also, the progress of regulation is incipient because there are scarce environmental public policies in connection with the Lagoon of Lobos, despite of representing an important economic source of funding provided by tourism.
\end{abstract}

Key Words: Environmental Law, Wetlands, Regulations, Sustainability, Ecosystems.

Sumario: I. Introducción. II. Metodología. III. Resultados y discusión. 3.1. El caso de Argentina en relación con la protección jurídica del ambiente y de los humedales. 3.2. Análisis sobre la normativa vigente sobre la protección de los humedales en otros países de América Latina. 3.3. El caso de estudio. El territorio del Partido de Lobos: Características generales y normativa municipal. 3.4. Las encuestas realizadas a la población urbana del Partido de Lobos. IV. Categorización en función de la sostenibilidad. V. Consideraciones finales. Bibliografía. 


\section{Introducción}

La Fundación Humedales de Argentina y Wetlands International definieron a los humedales como las áreas donde el agua se encuentra con la tierra, donde se acumula el agua o se inundan en algunos períodos, donde los animales y plantas que viven en estos ambientes están acostumbrados al agua, y que forman un ecosistema muy particular. Se encuentran entre los ambientes más productivos del mundo, llenos de biodiversidad y de servicios ecosistémicos, donde habitan diversas especies de flora y fauna, y además brindan importantes beneficios económicos y sociales a las personas. De ellos es posible obtener comida, agua, materiales para construcción y producción, que son fundamentales para quienes viven en su entorno.

Desde el punto de vista jurídico se define a los humedales de acuerdo a lo establecido en la Convención sobre los Humedales de Importancia Internacional conocida como Ramsar, que brinda una amplia protección ambiental y que establece que "son las extensiones de marismas, pantanos y turberas o superficies cubiertas de aguas, sean éstas de régimen natural o artificial, permanentes o temporarias, estancadas o corrientes, dulces, salobres o saladas, incluyendo las extensiones de aguas marinas cuya profundidad en marea baja no exceda los seis metros".

Argentina tiene actualmente veintitrés sitios Ramsar que abarcan un total de 5.684.525 hectáreas, que poseen una gran diversidad y biodiversidad. ${ }^{1}$

Por su parte, se seleccionará la categorización de tres clases de sostenibilidad: débil, fuerte y súper fuerte (Gudynas, 2011, p. 80).

En este contexto, para el análisis de la normativa que se mencionará posteriormente se utilizarán las categorías doctrinarias tomadas de Gudynas de acuerdo a los criterios que se detallarán a continuación.

\footnotetext{
${ }^{1}$ Puede accederse a más información en el siguiente enlace: https://www.argentina.gob.ar/ambiente/agua/ humedales/sitiosramsar
} 


\section{Cuadro 1}

En el caso de la normativa vigente en Argentina se destaca la incorporación del "enfoque ecosistémico", que justamente incluyó al "ecosistema" como unidad de gestión incluyendo a sus dimensiones naturales y socioculturales. Este fue definido en el año 2005 por la Evaluación de Ecosistemas del Milenio, como todos los beneficios que las poblaciones humanas obtienen de estos ámbitos, y que contribuyen a hacer la vida no sólo físicamente posible sino también digna de ser vivida (Costanza et al. 2007, Daily 1997).

En particular se destaca el artículo 5 de la Ley 26.331 de presupuestos mínimos de protección ambiental para el enriquecimiento, la restauración, conservación, aprovechamiento y manejo sostenible de los bosques nativos dictada en el año 2007. En la misma se reconoce a los servicios ambientales y los describe como "los beneficios tangibles e intangibles generados por los ecosistemas del bosque nativo, necesarios para el concierto y supervivencia del sistema natural y biológico en su conjunto, y para mejorar y asegurar la calidad de vida de los habitantes de la Nación beneficiados por los bosques nativos. Entre otros, los principales servicios ambientales que los bosques nativos brindan a la sociedad son: regulación hídrica, conservación de la biodiversidad, conservación del suelo y de calidad del agua, fijación de emisiones de gases con efecto invernadero, contribución a la diversificación y belleza del paisaje, y defensa de la identidad cultural. Esta norma jurídica que fue dictada en 2007 representa una de las que inicialmente ha incorporado esta categoría y enfoque en el Derecho Ambiental argentino y luego fue seguida por otras posteriores como la de glaciares (Ley 26.639 que fue dictada en el año 2010).

En el presente trabajo se propone, en primer lugar, realizar un relevamiento de la normativa aplicable al Partido de Lobos, en la Provincia de Buenos Aires, Argentina, para luego poder contrastarla con la legislación vigente en otros países de América Latina (Colombia, Costa Rica, Brasil, Perú, y Chile).

Posteriormente a dicho análisis normativo, se determinará dentro de qué categoría doctrinaria se encuentra la misma: sostenibilidad "super-fuerte", "fuerte" o débil", tal como ya se han descripto en el cuadro 1. En segundo lugar, se analizarán los resultados obtenidos en las encuestas realizadas a la población

\section{4}


del área urbana del Partido de Lobos y la información obtenida de entrevistas realizadas a informantes clave.

\section{Metodología}

Se ha recurrido a la estrategia de la triangulación metodológica. Se realizaron encuestas a la población urbana del Partido de Lobos, dos entrevistas a informantes clave de la zona y se utilizó la hermenéutica jurídica para el análisis de normativa. Específicamente se realizaron dos entrevistas a los informantes clave el día el 5 de septiembre de 2018 y cuyos datos se acompañan a continuación:

- Sara Castiglione, Magíster en Ingeniería en Calidad de la Universidad Tecnológica Nacional. Su tesis de maestría fue titulada: "Poder Judicial: indicadores de Gestión y Calidad como motor de mejora". Fue seleccionada para esta instancia debido a que posee experiencia académica y de campo en relación con el funcionamiento del Poder Judicial al haber realizado un análisis de sus fortalezas y debilidades desde el ámbito de la gestión de la calidad. - Laureano Della Schiava, Juez de Paz del Partido de Lobos, y nos fue referenciado por la anterior informante clave. Fue muy relevante su entrevista porque nos informó sobre el funcionamiento de su juzgado, la aplicación de la normativa y de los casos judiciales vinculados con la protección ambiental.

Además, se realizó un relevamiento de los niveles de interés y/o preocupación respecto de las cuestiones ambientales de la población en el ámbito del Partido de Lobos mediante el diseño, implementación y procesamiento de los resultados brindados por encuestas cuyas preguntas se detallarán a continuación. Estas últimas se realizaron personalmente y al azar, en donde en caso de no comprenderse alguna pregunta se aprovechó la interacción para poder explicarla adecuadamente.

Se trata de un muestreo no probabilístico en donde se realizaron encuestas a 33 personas que habitaban o trabajaban en el casco urbano del Partido de Lobos durante el mes de septiembre de 2018. Los criterios de selección se basaron en 
analizar una muestra poblacional con características y cantidades equitativas que sirvan para poder efectuar posteriores comparaciones. Los encuestados reunieron las siguientes características: nivel educativo: $25 \%$ tenían estudios primarios, $25 \%$ tenían estudios secundarios, $25 \%$ tenían estudios terciarios y $25 \%$ tenían estudios universitarios. A su vez en relación con el rango etario: 50\% de los encuestados tenían entre 18 y 40 años y el otro $50 \%$ tenía entre 41 y 70 años.

A todos los encuestados se les solicitó contestar por escrito un cuestionario con 22 preguntas vinculadas con diferentes temáticas ambientales, donde todas presentaron un formato de multiple choice. También se han realizado dos entrevistas a informantes clave.

\section{Resultados y discusión}

\subsection{El caso de Argentina en relación con la protección jurídica del ambiente y de los humedales}

En Argentina la incorporación del derecho al ambiente sano data del año 1994, momento en el que se reformó la Constitución Nacional de Argentina e incorporó el artículo 41 y el paradigma ambiental.

Debe tenerse en cuenta que en este país existe un sistema federal de gobierno, en donde las provincias conservan su autonomía a pesar de estar reunidas bajo un gobierno común (Gobierno Nacional). Sin embargo, estas últimas cedieron algunas de sus facultades, y es allí donde nos focalizaremos en el ámbito de la protección ambiental. En este sentido, el artículo 124 en su último párrafo establece que corresponde a las provincias el dominio originario de los recursos naturales existentes en su territorio. A su vez el artículo 123 establece que cada provincia dictará su propia constitución y deberá asegurar la autonomía municipal reglando su alcance y contenido en el orden institucional, político, administrativo, económico y financiero. 
Respecto a los humedales, no existe a nivel nacional y/o provincial, normativa referida a la protección de los mismos y de sus servicios ecosistémicos. Sin embargo, existe un proyecto de ley de presupuestos mínimos para la protección y uso racional y sostenible de los humedales de la Argentina, que fue iniciado en 2012. Para dicho proyecto trabajaron de manera conjunta desde la Fundación Humedales junto a legisladores, ONGs, universidades para apoyar la sanción de la ley en el Congreso de la Nación. A fines de 2013 se logró que el proyecto sea aprobado por unanimidad en el Senado de la Nación, pero el mismo no logró el apoyo suficiente en la Cámara de Diputados y perdió estado parlamentario a fines de 2015. Es por eso, que hasta el día de la fecha no se cuenta con legislación que busque conservar y proteger a estos ecosistemas. Sin embargo, en 2016 el gobierno de turno anunció la presentación de un proyecto de ley denominado "Presupuestos Mínimos para la Conservación, Protección y Uso Racional y Sustentable de los Humedales". El documento fue consensuado entre seis ministerios, entre ellos el de Ambiente, el de Energía, el de Interior y el de Obras Públicas.

En síntesis, no se ha dictado aún una ley nacional que regule la protección y la gestión de los humedales en Argentina atento a que diversos proyectos que se han presentado ante el Congreso de la Nación han perdido estado parlamentario y por lo tanto no han sido aprobados. Actualmente este debate se reanudó recientemente en Argentina debido a la quema sistemática de pastizales ubicados en el Delta. ${ }^{2}$

Sin embargo, este último proyecto de ley que finalmente no fue aprobado por el Congreso, en el cual se incorporaron particularidades específicas que presentan un enfoque ecosistémico superando al de la sostenibilidad. Esto se fundamenta en que en su artículo 2 se hace especial referencia a los conceptos de "integridad ecológica" y de "servicios ecosistémicos" que brindan los humedales, considerados como los beneficios tangibles e intangibles derivados de la estructura y funciones de estos ecosistemas.

Dentro de los objetivos de protección (artículo 3) se menciona a los procesos ecológicos y culturales de los humedales, a la biodiversidad, a la provisión del

\footnotetext{
2 https://www.pagina12.com.ar/283274-diputados-comenzo-a-debatir-la-ley-de-proteccion-de-humedale, https:// www.greenpeace.org/argentina/story/issues/climayenergia/greenpeace-mientras-el-humo-llega-a-buenosaires-crece-la-urgencia-por-una-ley-de-humedales/
} 
agua y a la regulación del régimen hidrológico, a las actividades para fomentar la restauración y a la implementación de medios de vida tradicionales o innovadores, "sostenibles" económica, social y ambientalmente en las áreas de los humedales.

Este proyecto de ley posee una estructura similar a la de las leyes de presupuestos mínimos para la protección de los bosques nativos 26.331 y de los glaciares 26.639 de Argentina, porque aquí también se ha creado un Inventario Nacional de Humedales. Sin embargo, la diferencia sustancial radica en que en este último caso el inventario deberá ser realizado de forma conjunta entre las autoridades provinciales y nacionales, para poder coordinar su elaboración y desarrollo y su actualización cada cinco años. En el caso de los bosques nativos se realizaron los ordenamientos territoriales por parte de las provincias, y en el de los glaciares por parte de un organismo nacional sin plantearse hasta el momento un trabajo conjunto entre las diferentes jurisdicciones.

Las provincias y el ámbito nacional (dentro de un proceso participativo) cuentan con dos años para realizar el inventario, y conjuntamente se deberá implementar el procedimiento administrativo de la evaluación de impacto y una evaluación ambientales estratégica.

Según este proyecto de ley, existen tres categorías de humedales: (artículo 15).

- “Área de preservación: Sectores de alto valor de conservación que no deben transformarse.

- Área de gestión de recursos: Sectores de humedales de medio valor de conservación, que admitan actividades productivas extensivas, que deberán ser gestionados para optimizar la provisión de los servicios ambientales de los humedales. Estas áreas admiten actividades de impacto moderado [...]. - Área de usos múltiples: Sectores donde actualmente se realizan actividades económicas o que tiene vocación productiva, pero que debieran realizarse incluyendo criterios de sostenibilidad."

Luego de dos años de la aprobación del proyecto de ley, se exige la elaboración del ordenamiento territorial de los humedales existentes en el país. Dicho instrumento deberá contener al menos tres escalas espaciales: nivel de regiones de humedales del país, de paisajes o de sistemas de humedales y de detalle local con unidades de humedales. Además, se deberá incorporar información sistematizada para 
su ubicación y actualizarse los cambios en las superficies, estados de avances o retrocesos, características ecológicas, entre otros aspectos.

Las sanciones por incumplimiento establecidas en este proyecto de ley son similares a las de otra normativa ambiental de Argentina mencionada, atento a que se imponen las de índole administrativa como es el caso de las multas, de los apercibimientos, entre otras, que no logran disuadir ni modificar conductas dañinas hacia el ambiente y que podrían considerarse débiles.

Dentro del ámbito de las políticas públicas podríamos mencionar a la elaboración del Inventario de humedales que fue mencionada anteriormente, el cual se encuentra publicado desde febrero de 2018 y que fue sostenido por la Secretaría de Ambiente y Desarrollo Sustentable y por investigadores de universidades públicas, como es el caso de la Universidad Nacional de San Martín, de la Universidad de Buenos Aires y de ONG's la Fundación Humedales (recibiendo también el apoyo de Ramsar). No se ha relevado ninguna otra acción de importancia vinculada con la protección de los humedales.

\subsection{Análisis sobre la normativa vigente sobre la protección de los humedales en otros países de América Latina}

Todos los países que se analizarán poseen una tradición constitucional de incorporación del paradigma ambiental o de reconocimiento de la protección del ambiente, por lo que partimos de un aspecto positivo en donde puede materializarse un desarrollo normativo posterior vinculado con estas temáticas. El análisis propuesto también tiene la función de servir para apreciar la experiencia de otros países de la región que poseen características sociales, ambientales, culturales y/o geográficas similares a las de Argentina.

A continuación, se exhibirán los aspectos más relevantes de los textos constitucionales de los artículos vinculados con la protección ambiental que pueden aplicarse para el caso de los humedales. 


\section{Cuadro 2}

Posteriormente se graficará el estado actual de la normativa en relación con la gestión y/o protección específica de los humedales en los países mencionados detectando que todas se encuentran incluidos dentro de la categoría de Decretos y de Resoluciones y que no existe una ley específica que posea una jerarquía superior.

\section{Gráfico 1}

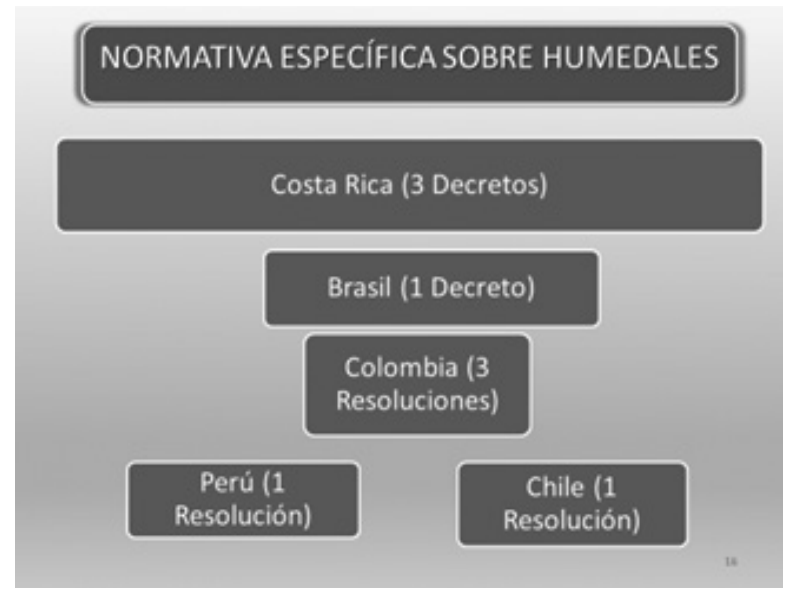

Elaboración propia, 2017

Debe destacarse que en los seis países analizados (Argentina, Colombia, Costa Rica, Brasil, Perú y Chile) se ha detectado una complementariedad existente entre dos tipos de normas jurídicas:

1) Las que regulan específicamente a los humedales, $y$

2) Las que regulan otros aspectos ambientales y no relativos a los humedales, pero que los mencionan dentro de su texto.

En particular, en todos estos países existe una jerarquía aplicada a las diferentes clases de normativa jurídica. 
En primer lugar, la Constitución Nacional en todos los casos es la ley suprema y respecto de la cual se detallarán posteriormente los principales artículos vinculados con la protección ambiental que podrían haber influenciado en el desarrollo de normativa específica sobre los humedales. En los países que poseen un sistema de gobierno federal (Argentina y Brasil), existen normas nacionales y provinciales cuyas jerarquías deberían analizarse por separado, y en el caso de los otros países que poseen un sistema de gobierno unitario (Colombia, Chile, Perú y Costa Rica) no debe tenerse en cuenta el aspecto anterior.

Luego en segundo lugar, jerárquicamente se encuentran las leyes, le siguen los decretos del Poder Ejecutivo que reglamentan a las anteriores para que puedan ser aplicadas en la práctica, luego se ubican las Resoluciones Ministeriales y finalmente las Disposiciones de órganos inferiores.

En el caso de Costa Rica se destacan tres decretos que hacen referencia específica a los humedales. El Decreto Ejecutivo 22.550-MIRENEM de 1993 declaró que son humedales todas las áreas de manglares adyacentes a los litorales continentales e insulares. El Decreto 35803-MINAET de 2010 fijó los criterios técnicos para la identificación, clasificación y conservación de humedales y establece que se consideran igualmente a los ecosistemas de humedales en tanto estén o no creados por decreto o por ley y un sistema de clasificación de humedales que fue propuesto por la Convención de Ramsar. El Decreto Ejecutivo 29342-MINAE de 2001 titulado "Requisitos para la Renovación de Permiso de Uso Existentes en Áreas de Manglar" establece que para toda renovación de permiso de uso existente en áreas de manglar relacionadas con la producción de sal o de camarones deberá presentarse un plan de manejo por parte del interesado.

El caso de Brasil presenta como normativa específica un solo Decreto, el $\mathrm{N}^{\circ}$ 14.273 dictado en 2015 que prevé la zona de uso restringido de los humedales del Pantanal en el Estado de Mato Grosso do Sul y otras medidas ambientales. Esta norma es muy breve, es escasa para lograr una adecuada protección de los humedales y deberá complementarse con otra específica.

En cambio, Colombia posee mayor desarrollo normativo, en donde la Resolución № 157 de 2004 reglamenta el uso sostenible, conservación y manejo de los humedales y se desarrollan aspectos referidos a los mismos en aplicación de la Convención Ramsar. La Resolución N¹96 de 2006 del Ministerio de Ambiente 
y Desarrollo Sostenible (MAVDT) adopta la guía técnica para la elaboración de planes de manejo para humedales para la formulación, complementación o actualización por parte de las autoridades ambientales competentes en su área de jurisdicción, de los planes de manejo para los humedales prioritarios y para la delimitación de los mismos. La Resolución $\mathrm{N}^{\circ} 1128$ de 2006 MAVDT que modifica el artículo 10 de la Resolución $\mathrm{N}^{\circ} 839$ de 2003 y el artículo 12 de la Resolución $N^{\circ} 157$ de 2004 establecen lo siguiente: “Artículo 12. El Plan de Manejo del Humedal elaborado con base en la guía técnica a que se refiere la presente Resolución, será aprobado por el Consejo o Junta Directiva de la respectiva autoridad ambiental competente". Esto es relevante porque se aplicará un criterio integral de protección ambiental de los humedales en lugar de un enfoque priorizandola productividad.

Para el caso de Chile se ha relevado una sola norma jurídica específica, la Resolución Nº 909 de 1996 que identifica y delimita las zonas que corresponden a acuíferos que alimentan áreas de vegas y de los llamados bofedales en las regiones de Tarapaca y de Antofagasta. La misma fue modificada y actualizada por la $N^{\circ} 464$ dictada en 2006 pero no desarrolla lineamientos relevantes.

En el caso de Perú existe una norma jurídica específica que posee la categoría de Resolución Ministerial 248-2015-MINAM que determina los lineamientos para la designación de sitios Ramsar y que coincide con el instrumento internacional.

\subsection{El caso de estudio. El territorio del Partido de Lobos: Características generales y normativa municipal}

En el Noreste de la Provincia de Buenos Aires se ha modificado el paisaje original de la pampa húmeda por el de los "ecosistemas agrícolas" y se han realizado cambios en el uso del suelo.

La agricultura es una de las principales actividades productivas en el área y es la primera fuente del mercado interno y de exportación del país. Allí en algunos casos existe una interacción ecosistema-hombre que es característica de los agroecosistemas, y por eso deben valorarse significativamente los servicios ecosistémicos que éstos proveen. En la actualidad la mayor parte de los mismos 
se encuentran dedicados al suministro de una única categoría de servicios, que son los de aprovisionamiento (Aznar y Velasco, 2016, p. 168).

En el caso de la Laguna de Lobos se trata de un ambiente que constituye un importante centro de actividades recreativas, turísticas y deportivas. Es una reserva en donde se protege flora y fauna autóctona del ecosistema humedal de la pampa bonaerense.

El afluente principal de la laguna es el Arroyo Las Garzas, colector principal de una cuenca de drenaje de $1720 \mathrm{~km}^{2} \mathrm{y}$ habitualmente recibe efluentes pecuarios, industriales y cloacales que confluyen en su cauce en el tramo comprendido entre la Laguna de Navarro y la Laguna de Lobos (Pozzobon, 2009, p. 186).

La densidad poblacional de este Partido es de aproximadamente 38.900 habitantes por lo que se considera una localidad pequeña, con características culturales y sociales similares.

Desde el ámbito del análisis normativo, se destaca el Decreto municipal $\mathrm{N}^{\circ} 931$ de 2011 que declaró de interés municipal la realización del Taller de Protección del Medio Ambiente para niños, y el Decreto municipal N 388 de 2012 que reglamenta la capacitación ambiental de docentes que fue dictada por el Organismo Provincial para el Desarrollo Sustentable. Del mismo año la Resolución municipal N 432 rechaza la ganadería, agricultura, y minería que atenten contra el ambiente.

El Decreto municipal $N^{\circ} 803$ de 2013 hace referencia al $3^{\circ}$ Encuentro de responsables ambientales, el Decreto municipal No 251 de 2017 se refiere a la figura de los ecodelegados, la Resolución municipal № 518 de 2017 menciona al programa educando Argentina medioambiente, y el Decreto municipal No 787 de 2017 describe el Programa “Arboleando". La Resolución municipal N 539 de 2018 hace lo mismo respecto de la producción agroecológica rural y urbana.

No se ha relevado normativa municipal ni provincial que se apliquen en la práctica y que se encuentren vinculadas específicamente con los humedales, pero si con aspectos ambientales en general, a pesar de tener uno de esos ecosistemas dentro de su territorio. Existen escasas políticas públicas ambientales vinculadas directamente con la protección de los humedales y de sus ecosistemas. Sin embargo, se registraron diversas iniciativas vinculadas con propuestas sobre educación ambiental, que fomentan la participación pública y que aumentan notablemente los niveles de conciencia y de protección desde lo local. 


\subsection{Las encuestas realizadas a la población urbana del Partido de Lobos}

Una de las razones que sustentaron la implementación de encuestas es que el ámbito espacial de análisis no involucró a ciudades grandes por lo que resulta interesante relevar si su población posee o no una relación más cercana con la naturaleza que los que habitan en grandes ciudades, debido a que se encuentran a una distancia relativamente cercana del área rural. Ese contacto y cercanía podría generar un mayor respeto por la biodiversidad, por la protección del ambiente y también de los servicios ecosistémicos, debido a que culturalmente la población se relaciona de manera más estrecha con estos. Además, a través de las encuestas se pretende comprobar cuál es el nivel de información, preocupación y percepción que posee la población sobre las normas jurídicas existentes en relación ambiental y su protección.

Para la siguiente sección se procesaron e incorporaron estos resultados en cuadros sinópticos y para su elaboración se tuvo en consideración la información solicitada de forma obligatoria a todos los encuestados: nivel de educación y edad.

\section{Cuadro 3}

El cuadro 3 presenta los resultados respecto a la pregunta: ¿Escuchó hablar de problemas ambientales en su ciudad?

Se detecta que existe un gran interés por los problemas ambientales de la ciudad (hasta un 83,3\% en los que tienen entre 31 y 40 años). En la población que posee mayores niveles de educación se registra más cantidad de respuestas positivas ( $100 \%$ en los que poseen estudios terciarios y $71,4 \%$ con universitarios). Por el contrario, se reduce el nivel de interés cuando la población posee solamente estudios primarios, ya que en un $100 \%$ manifiesta que no estaba interesado en las problemáticas ambientales de su ciudad.

\section{Cuadro 4}

El cuadro 4 presenta los resultados respecto a la pregunta: ¿Le preocupan los aspectos y/o problemas vinculados con el ambiente en su ciudad? 
Se detectan altos niveles de preocupación respecto de los aspectos y/o problemas ambientales en la ciudad, en tanto en la opción "mucho" se registra un $83,3 \%$ en los encuestados que poseen estudios terciarios, $82,4 \%$ en los que tienen secundarios, y $71,4 \%$ en los que poseen una carrera universitaria. En cambio, los encuestados con estudios primarios contestan en un $66,7 \%$ que se encuentran poco preocupados por dicha situación, y el 33,3\% que no registran en absoluto ningún interés sobre la problemática ambiental en su ciudad.

Se destaca que la población que tiene entre 51 y 60 años manifiesta que no le interesa el tema en un $17 \%$ de las respuestas.

\section{Cuadro 5}

El cuadro 5 presenta los resultados respecto a la pregunta: ¿Cuánto le interesa informarse sobre temas ambientales?

Nuevamente los encuestados con niveles más altos de educación (universitaria y terciaria respectivamente) manifiestan mayor interés, desde un 71,4\% hasta un $83,3 \%$. Asimismo, la población que tiene entre 31 y 50 años es la que refleja niveles más altos de interés, en informarse sobre las problemáticas ambientales llegando hasta un 100\% en la franja de 41-50 años.

\section{Cuadro 6}

El cuadro 6 presenta los resultados respecto a la pregunta referida a los niveles de importancia que le brinda la población a los temas ecológicos y al cuidado del ambiente.

Nuevamente los encuestados con niveles más altos de educación manifiestan que poseen mayor interés por dichas cuestiones llegando a un $83,3 \%$ en los que poseen estudios terciarios, en comparación con un 33,33\% que no lo considera relevante y otro $33 \%$ que no sabe o no contesta, y todos en estos últimos casos poseen únicamente estudios primarios.

La población que posee entre 31 y 50 años también refleja niveles más altos de interés, llegando hasta un $100 \%$ en la franja de 41 a 50 años. 


\section{Cuadro 7}

El cuadro 7 presenta los resultados respecto a la pregunta: ¿Considera que la participación de la población puede ser determinante para lograr una mayor protección del ambiente?

En la mayoría de los casos y en todas las categorías, la mayoría de los encuestados afirma que la participación de la población es muy relevante sin marcarse distinciones en el rango etario y en el nivel de educación.

\section{Cuadro 8}

El cuadro 8 presenta los resultados respecto a la pregunta: ¿Cree que está en sus manos el cuidado del ambiente para las generaciones futuras?

La mayor parte de los encuestados manifiesta que considera que dependía de su accionar el cuidado del ambiente y sus consecuencias para el futuro, pero esto se ve reflejado en las personas que tenían mayores niveles de educación (70,6\% nivel secundario, $66 \%$ terciario y el más alto con $85,7 \%$ para el universitario). A su vez fueron las generaciones más jóvenes las que se pronunciaron de la misma manera $(76,9 \%$ los que tenían entre 18 y 30 años, 83,3\% los que tenían entre 31 y 40 , y $66 \%$ los que tenían entre 41 y 50 ).

Se destaca que las personas que poseen únicamente estudios primarios afirman que su accionar no tenía relación con el cuidado del ambiente en un 100\% de los casos. También se relevan altos porcentajes en la opción de la respuesta "no sabe, no contesta", reflejando un gran desinterés por esta problemática.

\section{Cuadro 9}

El cuadro 9 presenta los resultados respecto a la pregunta: ¿Cuál de las siguientes actitudes refleja mejor su situación personal en relación con su intervención en la protección del ambiente?

La misma estuvo orientada a consultarle al encuestado respecto de su participación concreta en relación con la protección del ambiente. Dentro de esta misma se incluyeron a las acciones que forman parte de la vida cotidiana (por

216 DERECHO GLOBAL. ESTUDIOS SOBRE DERECHO Y JUSTICIA 
ejemplo, realizar la separación de residuos), como también actividades que se implementan en conjunto con alguna asociación civil u ONG ambiental.

En este caso fue notoria la reducida participación que admiten los encuestados tanto los que poseen estudios universitarios (14\%) como secundarios (17\%), y esta tendencia se refleja en todas las categorías etarias. En la mayoría de los casos los encuestados se orientan a contestar que "intentaban actuar", o sea que esto podría interpretarse como una situación intermedia entra otras dos posibles respuestas que se habían planteado, que sería la de que realmente participan de algo y de la que no saben qué hacer (con un $47 \%$ para los encuestados con estudios secundarios). Se destacan las personas que poseen únicamente estudios primarios en donde se registran las siguientes respuestas: "no sé qué hacer", "no me interesa actuar" y "no sabe, no contesta", todos con un 33,3\% cada una, y no se registra ninguna respuesta en las que establecen que participaban o que al menos intentaban hacerlo.

Las personas que poseen entre 61 y 70 años a su vez manifiestan que intentaban actuar (50\%), pero el otro $50 \%$ de los encuestados contesta que no sabían o directamente no contestan.

\section{Cuadro 10}

Finalmente, el cuadro 10 presenta los resultados respecto a la pregunta: ¿Cuáles medidas considera más eficaces para resolver los problemas ambientales de su ciudad y/o región?

Se destaca que los encuestados que poseen niveles más altos de educación se inclinan a contestar en algunos casos que era más recomendable desarrollar programas de educación ambiental y mejorar la aplicación de las leyes existentes (40\% para ambos casos de los que poseen educación terciaria).

Sin embargo, se resalta que el $45 \%$ de los encuestados que posee un nivel de educación universitario afirma que consideraba que la mejor medida era imponer multas, y un $33 \%$ del mismo segmento se orienta hacia la implementación de programas de educación ambiental.

Los encuestados que poseen un nivel de educación primaria contestan en un $25 \%$ que no sabían qué responder sobre este tema y fueron los únicos que lo hicieron respecto de todos los segmentos. 
A su vez, los encuestados de 18 a 40 años consideran entre un 50 y un $60 \%$ respectivamente que la mejor medida era la de imponer multas, pero a su vez los de 31 a 40 años en un $40 \%$ afirman que también se inclinaban por la propuesta de desarrollar programas de educación ambiental.

\section{Categorización en función de la sostenibilidad}

Luego de haberse descripto los principales aspectos de las Constituciones Nacionales y de la normativa interna vinculada con la gestión y protección ambiental de los humedales de Argentina, Costa Rica, Brasil, Chile, Colombia y Perú, es posible aproximarse a establecer algunas clasificaciones vinculadas con las nociones doctrinarias esgrimidas anteriormente que se vinculan con la sostenibilidad. Esto se fundamenta en que en la práctica es casi imposible separar aspectos técnicos o de protección jurídica en este caso de los políticos que definen los niveles o matices aplicables en relación con la sostenibilidad.

A los fines del presente trabajo se ha clasificado a la normativa dentro de las diferentes clases de sostenibilidad de la manera que se describirá a continuación.

\section{Cuadro 11}

En el caso de la Constitución Nacional de Argentina, conforme surge del texto del artículo 41 puede catalogarse dentro del grupo de la "sustentabilidad débil", en tanto se establece que el ambiente sano debe estar en condiciones para que pueda desarrollarse la población teniendo en consideración las necesidades de las generaciones futuras. El daño ambiental generará la obligación de recomponer el ambiente (retrotraerlo a las mismas condiciones en que se encontraba antes de producirse el daño ambiental), y si esto no fuera posible se deberá reparar económicamente a través del pago de una indemnización pecuniaria.

La Constitución de Costa Rica puede clasificarse dentro de la categoría doctrinaria de la "sostenibilidad débil", debido a que se limita el ambiente o la naturaleza al desarrollo de las actividades productivas. 
Chile y de Brasil poseen dos artículos constitucionales referidos a la presente temática y plantean aspectos vinculados con la "sostenibilidad fuerte" porque en los mismos se aspira a lograr un ambiente ecológicamente equilibrado y a la preservación de la naturaleza.

El artículo 24 de la Constitución Nacional de Brasil avanza aún más, en tanto establece que debe considerarse también dentro del ambiente al patrimonio histórico, cultural, turístico y paisajístico y a los derechos y valores artísticos, estéticos.

Para Colombia se plantean también dos categorías doctrinarias debido, a que en el artículo 79 se incorporó a la población como sujeto fundamental en relación con la protección del ambiente (sostenibilidad súper fuerte), y en el artículo 80 se destacó la "sostenibilidad débil" debido a que se mencionó al desarrollo sostenible, pero en relación con la imposición de sanciones legales y al exigir la reparación de los daños ambientales ocasionados. Lo cuestionable es que en este país se ha regulado la temática a través de resoluciones que se encuentran en un nivel bajo de la pirámide legal.

En la carta magna de Perú todos sus artículos aportan aspectos vinculados con una "sostenibilidad débil" y se hace referencia a los recursos naturales como patrimonio del Estado y como soberano en su aprovechamiento destacando a las concesiones como herramienta fundamental para su uso.

Luego, en relación con la normativa ya analizada y vinculada a la protección de los humedales se han relevado situaciones dispares.

El proyecto de ley que se encontraba en trámite ante el Congreso Nacional de Argentina y que fue mencionado en el apartado 3, de acuerdo a sus características podría incluirse dentro de la categoría doctrinaria de "sostenibilidad super fuerte" atento a que hace referencia a los procesos ecológicos y culturales. A su vez, también incorpora herramientas vinculadas con la sostenibilidad débil, como es el caso de las evaluaciones de impacto ambiental y de las evaluaciones ambientales estratégicas que son funcionales principalmente a los intereses vinculados con la implementación de actividades productivas.

En el caso de Costa Rica una serie de decretos regulan la cuestión estableciendo aspectos relacionados con la categoría doctrinaria de la "sostenibilidad débil" porque la jerarquía de la normativa es inferior a las 
leyes, reproduce lo establecido en la Convención de Ramsar y se enfoca en el aprovechamiento productivo de los humedales.

En Brasil y en Colombia los decretos que regulan esta protección jurídica están incorporados en la categoría de "sostenibilidad súper fuerte", mientras que para Chile y Perú se encuentran dentro de la de "sostenibilidad débil" a través del dictado de Resoluciones que revisten una categoría normativa baja.

A continuación, se presentará un cuadro donde se clasificarán dentro de los tres niveles de sostenibilidad a los resultados obtenidos en las ocho preguntas de las encuestas.

Debido a que la mayoría de la normativa regional se focaliza únicamente en la gestión y/o protección de un recurso en particular, esto dificulta que se puedan incorporar los enfoques ecosistémicos dentro de la misma (Minaverry, 2017).

\section{Cuadro 12}

En el cuadro anterior se destaca que las primeras seis preguntas realizadas a los encuestados tienen un enfoque teórico, en donde se les consultó respecto de sus posiciones personales o de su entorno en relación con las problemáticas ambientales. Los porcentajes más altos se vinculaban con los lineamientos establecidos por la "sostenibilidad fuerte", debido a que en todos los casos manifestaron que su prioridad se focalizaba en la protección del ambiente y en la defensa de la participación pública.

Luego en las dos últimas preguntas se relevó el nivel de participación de la población en cuestiones ambientales, y las clases de sanciones/medidas que aplicarían para lograr una mayor protección ambiental, y las respuestas de los encuestados se orientaron hacia la "sostenibilidad débil". La mayoría estableció que la aplicación de multas era la mejor solución para resolver los problemas ambientales de la ciudad en lugar de la implementación de programas de educación ambiental, donde se podrían incorporar lineamientos vinculados con el enfoque ecosistémico brindado por la "sostenibilidad fuerte". Esta última categoría doctrinaria no ha sido detectada en las preguntas de las encuestas debido a que ninguna planteó su enfoque porque no se relevaron aspectos culturales.

\section{DERECHO GLOBAL. ESTUDIOS SOBRE DERECHO Y JUSTICIA}


Sin embargo, al haber realizado las dos entrevistas a los informantes clave fue posible constatar que no se han iniciado causas ambientales ni realizado denuncias relevantes ante el ámbito de la justicia en el Partido de Lobos que se vinculen con la protección de los humedales (hasta el 5 de septiembre de 2018).

\section{Consideraciones finales}

En los seis países analizados se ha relevado normativa específica vinculada con la protección de los humedales, siendo que alguna pertenece a las jerarquías inferiores de la pirámide jurídica como es el caso de los Decretos y de las Resoluciones. En todos los casos no se ha dictado ninguna ley sobre la temática, lo que podría estar indicando un escaso interés, falta de afianzamiento en las agendas políticas locales y/o la reducción de presupuestos económicos para lograr su aplicación en la práctica.

Además, la mayoría de los países analizados comparten los siguientes aspectos:

- Un marco normativo internacional, el Convenio Relativo a Humedales de Importancia Internacional, especialmente como hábitat de Aves Acuáticas (Ramsar) que fue suscripto por todos $\mathrm{y}$ reproducido por algunas normativas internas. - Escasas políticas públicas ambientales en relación con la protección de los humedales. - La mayoría de las Constituciones Nacionales poseen un enfoque de sostenibilidad débil, y lo mismo ocurre para el caso de la normativa vinculada con la protección de los humedales. Respecto del caso de estudio se destaca:

-Undesarrollo normativo municipal escaso en tanto no existe normativa nipolíticas ambientales específicas vinculadas con la Laguna de Lobos, a pesar de que su protección contribuye a mantener una fuente relevante de financiamiento económico proveniente del turismo. - La débil participación de la sociedad civil en la conservación, en el manejo de los humedales y en la protección del ambiente en general. - Se detectaron incipientes iniciativas municipales y al menos en este estudio no ha podido encontrarse ninguna relación específica entre los niveles de interés o de preocupación 
sobre los asuntos ambientales declarados por parte de la población y un mayor diseño e implementación de políticas públicas ambientales vinculadas con la protección de los humedales.

En este sentido y luego de haberse realizado entrevistas a informantes clave se puede afirmar que todos los actores sociales presentan especial interés en los resultados de las encuestas realizadas a la población, en tanto en algunos casos son consideradas como un móvil para diseñar o implementar políticas públicas o dictar o proponer normativa ambiental. Respecto del análisis de los resultados de las encuestas realizadas a la población es posible destacar que los niveles de educación como las edades de los encuestados marcan algunas tendencias cuyo análisis resulta interesante y se destaca que a menores niveles de educación se muestra un reducido interés por las problemáticas ambientales de su región.

A su vez, se relevó que los encuestados más jóvenes se encuentran más preocupados e informados sobre los asuntos ambientales que los más grandes.

\section{Bibliografía}

Aznar Sánchez, J. y Velasco Muñoz, J. (2016). Valoración de los ecoservicios en los agrosistemas españoles: un estado de cuestión. Revista Observatorio Medioambiental, (19), Anuario 2016, Madrid, Ediciones Complutense, pp. 165-180. Recuperado de https://revistas. ucm.es/index.php/OBMD/article/view/54166

Costanza et al. (1997). The value of the world's ecosystem services and natural capital. Nature, volumen 387, pp. 253-260.

Daily, G.C. (1997). Nature's Services: societal dependence on natural ecosystems. Island Press, pp. 392.

Gudynas, E. (2011). Desarrollo y sustentabilidad ambiental: diversidad de posturas, tensiones persistentes, en Matarán Ruíz, A y López Castellano F. (editores), La Tierra no es muda: diálogos entre el desarrollo sostenible y el postdesarrollo (pp. 69-96). Granada: Editorial Universidad de Granada. 
Minaverry, C. (2017). ¿Avances o retrocesos?. La evolución de los paradigmas sobre gestión ambiental en relación con la normativa y jurisprudencia sobre servicios ecosistémicos en América Latina. Revista Lex Social (Revista de derechos sociales), vol 7, $\mathrm{N}^{\circ}$ 1, pp. 476-493,

Pozobón, M. V. (2009). Descripción ambiental de la Laguna de Lobos, Pcia. Bs. As. Una propuesta para su monitoreo. Revista Biología Acuática (26), pp. 185-195. Recuperado de http://sedici.unlp.edu.ar/ handle/10915/68791

Como citar el artículo: Minaverry C, (2020). ¿Sostenibilidad fuerte o débil? Perspectivas de la protección jurídico- ambiental y ecosistémica de los humedales. El caso del Partido de Lobos, Provincia de Buenos Aires, Argentina. Derecho Global, Estudios sobre Derecho y Justicia, VI (16) pp. 201-223 https://DOI.org/10.32870/dgedj.v6i16.350 
